\title{
NEUTRON SPECTRUM PARAMETERS IN TWO IRRADIATION CHANNELS OF THE NIGERIA RESEARCH REACTOR-1 (NIRR-1) FOR USE IN $K_{0}$-NAA
}

\author{
*Sadiq, U. ${ }^{1}$ Jonah, S.A. ${ }^{2}$ Nasiru, R. $^{1}$ and Zakari, Y.I. ${ }^{1}$ \\ ${ }^{1}$ Department of Physics, Ahmadu Bello University, Zaria \\ ${ }^{2}$ Reactor Engineering Section, Centre for Energy Research and Training, Ahmadu Bello University, Zaria \\ Correspondence author: sadiqumarx@yahoo.co.uk
}

\begin{abstract}
For the utilization of the NIRR-1 to include the $k_{0}$-standardization method, the following neutron spectrum parameters in one inner and one outer irradiation channels were determined using the "Cd-ratio for multi-monitor method": The thermal to epithermal flux ratio, $f$, and the epithermal flux shape factor, $a$. These parameters determined in the inner irradiation channel $A_{1}$ are: $a=-$ $0.046 \pm 0.005$ and $f=18.4 \pm 0.34$ and for the outer irradiation channel $A_{2} a=+0.024 \pm 0.002$ and $f=49.5 \pm 0.96$. The results compare well with reported values obtained from other reactor facilities with similar core configuration such as the Slowpoke and Miniature Neutron Source Reactor facilities available in literature.
\end{abstract}

Keywords: Neutron spectrum parameters; $k_{0}-N A A ; C d$-ratio for multi-monitor method; NIRR-1; Thermal to epithermal flux ratio; Epithermal flux shape factor.

\section{INTRODUCTION}

The Nigeria Research Reactor-1 (NIRR-1) is specifically designed for use in Neutron Activation Analysis (NAA), thus there is the need for a careful and complete characterization of the neutron flux parameters in the irradiation channels in order to optimize its utilization for NAA via relative, absolute and single comparator methods. Stable neutron flux characteristics are exhibited by low-power research reactors, such as, the NIRR-1, the Canadian SLOWPOKE and the Chinese MNSR, which are suitable for NAA via the $k_{0}$-standarndized method (Kennedy et. al., 2000, Akaho et al, 2002 and Acharya et al, 2003). The neutron spectrum parameters needed for the calculation of concentration based on the $k_{0}$ standardization method via the Hogdahl convention for the so-called " $1 / V$ " nuclides are: the thermal to epithermal ratio, $f$ and the shape factor of the epithermal neutron flux, $\alpha$, approximated by a $1 / \mathrm{E}^{1+\alpha}$ distribution neutron flux. The knowledge of $\alpha$ is essential for the correction of resonance integrals $I_{0}$, i.e. for the conversion of $I_{0}$ values to $I_{0}(\alpha)$ values for its use in actual $1 / \mathrm{E}^{1+\alpha}$ epithermal neutron spectrum. Much attention is given to the determination of $f$ and $\alpha$, since these two parameters vary from one reactor to another, as well as among the different irradiation sites in a given reactor. The experimental methods for the determination of neutron flux parameters required in both the Hogdahl convention and the Wescott formalism have been enumerated in several published works (De Corte et al, 1993). However, the "Cd-ratio for multi-monitor" method, for reactors with stable neutron flux, has been recommended for neutron flux monitoring. Moreover, the method has been adjudged the most accurate for $\alpha$-monitoring (De Corte et al, 1981). In this investigation, using Hogdahl convention for the so-called " $1 / V$ " nuclides, we have determined the neutron spectrum parameters (i.e. $f$ and $\alpha$ ) in one inner and one outer irradiation channel of NIRR-1 for application in $k_{0}$-standardized NAA method.

\section{MATERIALS AND METHODS}

In the "Cd-ratio for multi-monitor" method for the determination of $f$ and $\alpha$ (measure of the deviation of epithermal neutrons from the ideal 1/E distribution), the parameters are defined as

$$
f=Q_{0, i} \cdot(\alpha) \cdot\left(F_{C d, i} \cdot R_{C d, r}-1\right) \cdot \frac{G_{e, i}}{G_{t h, i}}
$$

where $\mathrm{i}$ is the monitor with well known $Q_{0}$-value.

A set of $\mathrm{N}$ monitors is irradiated with and without Cd cover, and the induced activities are measured with a detector. With all the monitors obeying the $\sigma(v)$ versus $1 / v$ dependence, $\alpha$ is the slope of the straight line plot of

$$
\log \frac{\bar{E}_{r, i}^{\alpha}}{\left(F_{C d .} R_{C d, i}-1\right) Q_{0, i}(\alpha) G_{e, i} / G_{t h, i}} \quad \text { versus } \log \mathrm{E}_{\mathrm{r}, \mathrm{i}}
$$

Mathematically, by using an iterative procedure for a set of $\mathrm{N}$ monitors, Eq. (2) can be rewritten as Eq. (3) and the $\alpha$-value is found as the root of the equation. 
Bajopas Volume 3 Number 1 June 2010

$$
\begin{gathered}
\alpha+\frac{\sum_{i=1}^{N}\left[\left(\log \bar{E}_{r, i}-\frac{\sum_{i=1}^{N} \log \bar{E}_{r, i}}{N}\right)\left(\log \frac{E_{r, i}^{\alpha}}{\left(F_{C d, i} R_{C d, i}-1\right) Q_{0, i}(\alpha) G_{e, i} / G_{t h, i}}-\frac{\left.\sum_{i=1}^{N} \log \frac{E_{r, i}^{\alpha}}{\left(F_{C d, i} R_{C d, i}-1\right) Q_{0, i}(\alpha) G_{e, i} / G_{t h, i}}\right)}{N}\right)\right]}{\sum_{i=1}^{N}\left(\log \bar{E}_{r, i} \frac{\sum_{i=1}^{N} \log \bar{E}_{r, i}}{N}\right)^{2}}=0 \\
\text { where, } \quad R_{C d}=\frac{A_{s p . b a r e}}{A_{s p . C d}} ; \quad A_{s p}=\left[\frac{N_{p} / t_{m}}{w S D C}\right]
\end{gathered}
$$

and

$$
Q_{0}(\alpha)=\left[\frac{Q_{0}-0.429}{\left(\bar{E}_{r}\right)^{\alpha}}+\frac{0.429}{(2 \alpha+1)\left(E_{C d}\right)^{\alpha}}\right](1 e V)^{\alpha}
$$

where $\mathrm{i}$ denotes the $\mathrm{i}^{\mathrm{t}}$ monitor, $\mathrm{N}$ the number of monitors used, $E_{r, i}$ the effective resonance energy of the $i^{\text {th }}$ monitor, $F_{c d}$ the Cd-transmission factor for epithermal neutrons, $G_{\text {eii }}$ the epithermal neutron selfshielding factor for the $i^{\text {th }}$ monitor, $G_{t h, i}$ the thermal neutron self-shielding factor for the $i^{\text {th }}$ monitor, $R_{c d, i}$ the ratio of the specific activity of the $i^{\text {th }}$ monitor irradiated without $\mathrm{Cd}\left(\mathrm{A}_{\mathrm{sp} \text {-bare }}\right)$ to that with $\mathrm{Cd}$ cover $\left(\mathrm{A}_{\mathrm{spCd}}\right)$ and $Q_{o, i}=I_{d} / \sigma_{o}$ the ratio of resonance integral to thermal neutron capture cross section at neutron velocity of $2200 \mathrm{~m} / \mathrm{s}$ for the $\mathrm{i}^{\text {th }}$ monitor.

\section{Experimental}

The NIRR-1 consists of five small inner rabbit tubes, which are installed inside the beryllium annulus. Between the beryllium annulus and the reactor vessel, three small and two large rabbit tubes are installed. They are designed as the outer irradiation channels. Out of a total of ten irradiation channels of NIRR1, six are connected to the pneumatic transfer systems. There are two sets of transfer systems; the pneumatic transfer system type $A$, which is connected to two of the irradiation channels, $A_{1}$, (small, inner channel) and $A_{2}$ (large, outer channel) is suitable for handling middle and long-lived nuclides. The other is a multifunctional transfer system, the pneumatic transfer system $B$. This is connected to three small inner irradiation channels $\left(B_{1}, B_{2} \& B_{3}\right)$ and a small outer irradiation channel $B_{4}$. System $B$ can be used to send a sample into the reactor and retrieve it to a specified position such as detector, stripper or waste tank. It is especially designed for NAA of short-lived nuclides and is suitable for cyclic NAA of nuclides with half-lives in seconds.

In this work, an inner irradiation channel $\left(A_{1}\right)$ and an outer irradiation channel $\left(A_{2}\right)$ were selected as representatives of all the irradiation channels. For the determination of $f$ and $\alpha$ values, the "Cd-ratio for multi-monitor" method was used because of its suitability for a priori $f$ and $\alpha$ monitoring in reactors with stable neutron spectrum parameters such as MNSR and SLOWPOKE facilities. Based on four monitor reactions, detector foils will be prepared from high-purity metals/alloys, which are recommended for neutron spectrum monitoring in reactor irradiation channels. Two sets of detector foils will be prepared for each of the irradiation channels. One set will be arranged in a stack inside a vial for the 'bare' irradiation and the second set arranged in a stack inside $1 \mathrm{~mm}$ thick Cd-cover and kept inside the vial for the Cd-cover irradiation.

In channel $A_{1}$, the irradiation was carried out for 30minutes for the 'bare' and 1 hour for the Cdcovered, while for channel $A_{2}$, the outer irradiation channel, the irradiation time was increased by a factor of 2 since the neutron flux in the outer channel is half the value in the inner channel. All irradiations were performed at a thermal power level of $15.5 \mathrm{~kW}$, which corresponds to a preset neutron flux of $5.0 \times 10^{11}$ $\mathrm{n} / \mathrm{cm}^{2} \mathrm{~s}^{-1}$ on the control console.

After the irradiation, the induced activities in the detector foils were measured on a GEM-300195 HPGe coaxial, vertical dip-stick detector (EG\&G ORTEC), which has a relative efficiency of $30 \%$ and resolution of $1.95 \mathrm{keV}$, at $1.33 \mathrm{MeV},{ }^{60} \mathrm{Co}$. The gamma-ray acquisition system consists of MAESTRO multi-channel Analyzer (MCA) emulation software card, coupled to the detector via electronic modules, all manufactured by EG\&G ORTEC. The multi-purpose gamma ray analysis software, WinSPAN-2004 developed by Wang Liyu (2004) was used for peak identification and evaluation. . For peak analysis of ${ }^{69 \mathrm{~m}} \mathrm{Zn}$ and ${ }^{198} \mathrm{Au}$ nuclides, the irradiated foils were counted within a day after appropriate cooling time at a source-detector distance of $5 \mathrm{~cm}$ to eliminate errors due to coincidence losses. For peak analysis of ${ }^{65} \mathrm{Zn}$ and ${ }^{95} \mathrm{Zr}$ radionuclides, irradiated foils were counted at least one week after irradiation at a close sourcedetector distance of $1 \mathrm{~cm}$.

Table 1: Description of the monitor foils used.

\begin{tabular}{llll}
\hline Monitor & Material description & Diameter, $\mathbf{c m}$ & Range of mass, mg \\
\hline $\mathrm{Au}$ & $\mathrm{Al}-0.1 \%$ Au; 0.1mm thick, IRMM-530 & 0.8 & $12-14$ \\
$\mathrm{Zn}$ & $99.95 \%$ Zn foil, 0.025mm thick Goodfellow. & 0.8 & $4-5$ \\
$\mathrm{Zr}$ & $99.8 \%$ Zr foil; $0.125 \mathrm{~mm}$ thick, Goodfellow & 0.8 & $44-46$ \\
\hline
\end{tabular}


Table2: Calculated values of $R_{\mathrm{Cd}}$ for NIRR-1 and nuclear data of detector monitors.

\begin{tabular}{|c|c|c|c|c|c|c|c|}
\hline $\begin{array}{l}\text { Target } \\
\text { nucleus }\end{array}$ & Product nuclide & $\begin{array}{l}\text { Half } \\
\text { life }\end{array}$ & $\mathbf{E}_{\mathbf{v}}, \mathbf{k e V}$ & $\bar{E}_{\gamma} \mathbf{e V}$ & $\mathbf{Q}_{0}$ & Inner & $\begin{array}{l}\mathbf{R}_{\text {cd }} \\
\text { Outer }\end{array}$ \\
\hline${ }^{197} \mathrm{Au}$ & ${ }^{198} \mathrm{Au}$ & $2.695 d$ & 411.8 & 5.65 & 15.7 & $2.10 \pm 0.01$ & $4.32 \pm 0.03$ \\
\hline${ }^{68} \mathrm{Zn}$ & ${ }^{69 m} \mathrm{Zn}$ & $13.76 \mathrm{~h}$ & 438.6 & 590.0 & 3.19 & $5.48 \pm 0.06$ & $18.64 \pm 0.35$ \\
\hline${ }^{94} \mathrm{Zr}$ & ${ }^{95} \mathrm{Zr}$ & $244.0 d$ & 115.5 & 2560 & 1.908 & $3.38 \pm 0.09$ & $12.53 \pm 0.52$ \\
\hline${ }^{64} \mathrm{Zn}$ & ${ }^{65} \mathrm{Zn}$ & $64.02 d$ & $\begin{array}{l}724.2 \\
+756.7\end{array}$ & 6260.0 & 5.36 & $8.28 \pm 0.29$ & $30.97 \pm 1.95$ \\
\hline
\end{tabular}

\section{RESULTS AND DISCUSSION}

The measured cadmium ratios in Table 2 of the three flux monitors used in the determination of $f$ and $a$ were determined from the ratio of specific activity, $A_{s p}$ of foil irradiated without to that irradiated with $\mathrm{Cd}$-cover. The $\mathrm{R}_{\mathrm{Cd}}$ values were then used to determine $f$ - and $a$-values by solving Eqs. (1) and (3) using an iterative procedure based on MS-EXCEL spreadsheet. Similarly, the values were also obtained from the plot of Eq. (2) versus $\log E_{r, I}$ as displayed in figs. 1 and 2 . The values of $f$ and $a$ obtained for NIRR-1 are given alongside reported values obtained from other MNSR reactors with similar core configuration in table 3 . The results indicate that the inner channels of the MNSR reactors are poorly thermalized (indicated by the negative a values) in comparison with the outer channels. This is to be expected because of the compact nature of the reactor core, designed with relatively low critical mass and low value of $\mathrm{H} /{ }^{235} \mathrm{U}$ atoms in the fuel lattice (i.e., core under moderation). This makes shimming effective and avoids frequent reloading of fuel elements as well as obtain a reasonably high value of thermal neutron flux in the Be annulus for NAA. The measured $a$ value, for the inner irradiation channel of NIRR-1, compares very well with the values for Slowpoke-2 reactors, but falls short by about $56 \%$ when compared with that of GHARR-1. The $f$ and $a$ values for the outer irradiation channel compare well with values of other MNSR and Slowpoke facilities presented in Table 3. This confirms the assertion that reactors with similar fuel have identical neutron spectra. The slight deviation of our a-value from the one reported by Jonah et. al.,2005 for NIRR-1 could be attributed to the channel location with respect to the core and effects of moderation, which could lead to slight differences in spectral indexes between irradiation channels. This is one of the reasons for the recommendation that the epithermal flux-shaping factor $a$, be measured for each irradiation channel (De Corte et al., 1981).

\section{CONCLUSIONS}

The characterization of the neutron flux spectra in one inner and one outer irradiation channels (A1 and $A 2)$ of the NIRR-1, for the purpose of adopting the $k_{0}$ standardized NAA method in our facility, was carried out and the results are in good agreement with previously reported values for irradiation sites B2 and B4 reported by Jonah et al, 2005. The values determined are also in agreement with those reported from other MNSR's with similar core configurations except for the deviation in the a-value for the inner irradiation channel of GHARR-1.

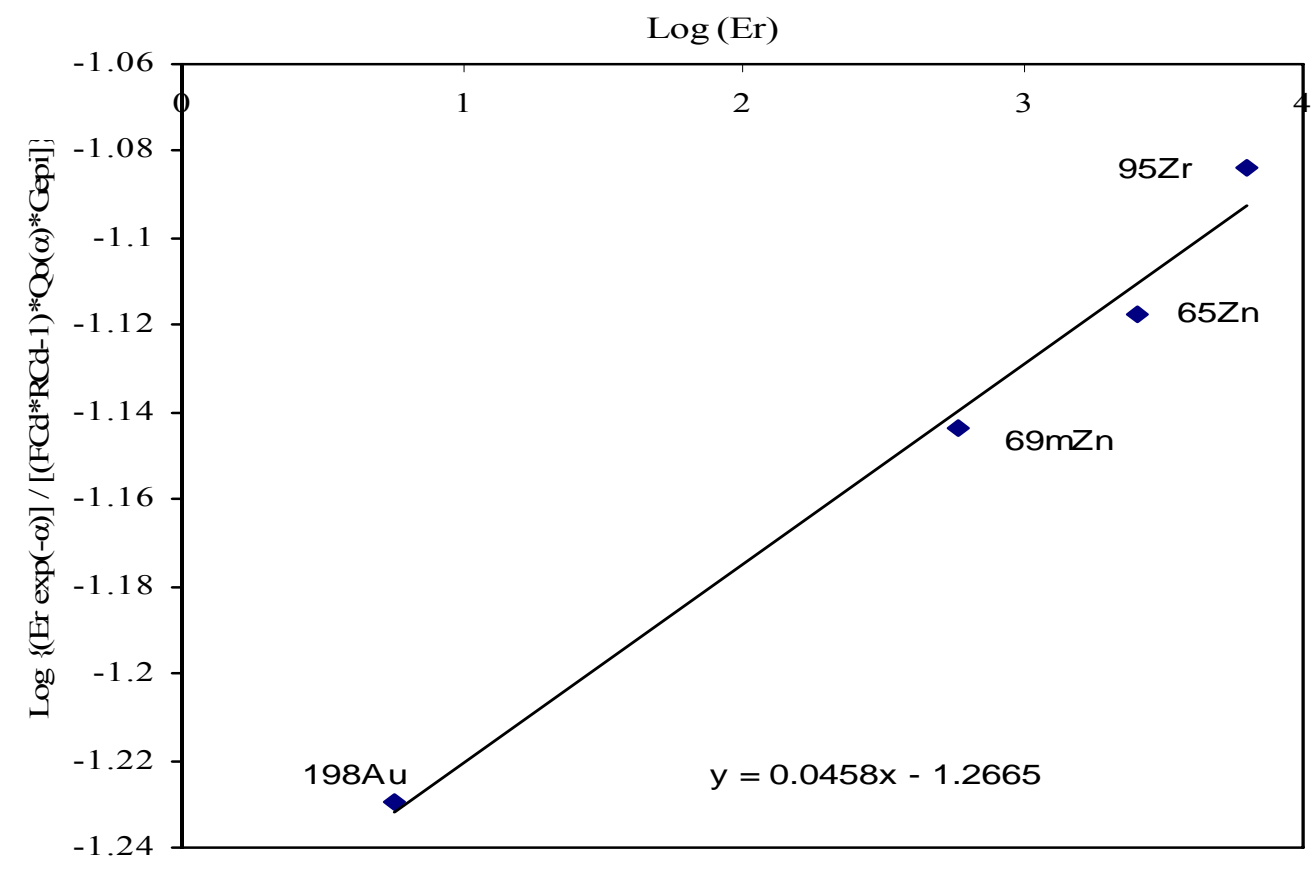

Fig. 1. Plot for the determination of $\mathrm{f}$ and $\alpha$ by "Cd Ratio for multi monitor" method for an inner irradiation Channel A1, of NIRR-1 


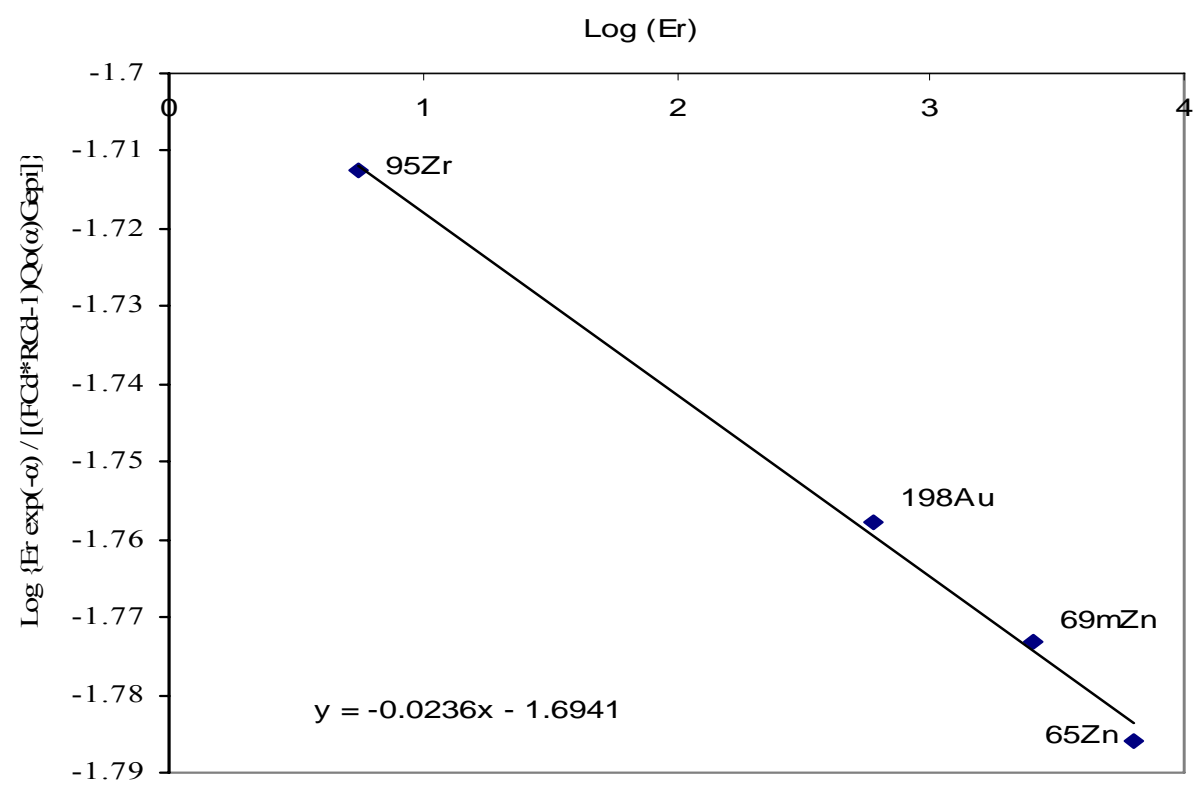

Fig. 2 Plot for the determination of $\mathrm{f}$ and alpha by "Cd Ratio multi monitor" method for an outer irradiation Channel, A2 of NIRR-1

Table 3: Comparison of $f$ and a values in irradiation channels of Slowpoke and MNSR facilities Facility

\begin{tabular}{|c|c|c|c|c|}
\hline & f & $\mathbf{a}$ & $f$ & $a$ \\
\hline $\begin{array}{l}\text { MNSR, GHARR-1; Ghana } \\
90 \%{ }^{235} \mathrm{U} \text {. }\end{array}$ & 18.8 & -0.104 & 49 & -0.0261 \\
\hline $\begin{array}{l}\text { MNSR, Prototype, Beijing, China, } \\
90 \%{ }^{235} \mathrm{U}\end{array}$ & 19.8 & -0.009 & 58.5 & +0.023 \\
\hline $\begin{array}{l}\text { Slowpoke-2,DUSR,Halifax Canada, } \\
20 \%{ }^{235} \text { U (site } \# 2 \text { ) }\end{array}$ & 18.8 & -0.0425 & 57.1 & -0.0098 \\
\hline $\begin{array}{l}\text { Slowpoke-2,DUSR,Halifax Canada, } \\
20 \%{ }^{235} \text { (site \#3) }\end{array}$ & 18.9 & -0.0422 & 57.1 & -0.0098 \\
\hline $\begin{array}{l}\text { Slowpoke-2,Ecole polytechnic } \\
\text { Montreal, Canada, } 20 \%{ }^{235} \mathrm{U}\end{array}$ & 19.8 & -0.052 & 55.4 & -0.006 \\
\hline $\begin{array}{l}\text { MNSR, }_{\prime} \text { NIRR-1; Zaria, Nigeria, } \\
90 \%{ }^{235} \cup \text { (sites B2 \& B4 ) }\end{array}$ & 19.2 & -0.052 & 48.3 & +0.029 \\
\hline $\begin{array}{l}\text { MNSR, NIRR-1; Zaria, Nigeria, } \\
90 \%{ }^{235} \mathrm{U} \text { (sites } \boldsymbol{A 1} \& \boldsymbol{A 2} \text { ) }\end{array}$ & $18.4 \pm 0.34$ & $-0.046 \pm 0.005$ & $49.5 \pm 0.96$ & $+0.024 \pm 0.002$ \\
\hline
\end{tabular}

\section{REFERENCES}

Acharya, R. N., Chatt, A. (2003): Determination of neutron flux parameters of dalhouise SLOWPOKE-2 for ko-NAA using Hogdahl convention and modified Wescott-formalism. Journal of Radioanalytical Chemistry, vol. 257, pp525

Akaho, E.H.K.; B.J.B. Nyarko.; (2002): Characterization of neutron flux spectra in irradiation sites of MNSR reactor using Westcott-formalism for the $\mathrm{k}_{0}$ neutron activation analysis method. J. Appl. Rad and Isotop. Vol. 57 pp265-273.

De Corte F.; Hammami, K. S.; Moens, L.; Simonits, A.; De Wispelaere, A.; Hoste, J.; (1981): The accuracy of the experimental a-determination in the $1 /\left(\mathrm{E}^{1+a}\right)$ epithermal reactor neutron spectrum. Journal of Radioanalytical Chemistry, vol. 62, pp209-255.

De Corte F.; Simonits, A.; Bellemans, F.; Freitas, M.C.; Javanovic, S.; Smodis, B.; Erdtman, G.; Petri, H.; De Wispelaere, A.; (1993): Recent advances in $\mathrm{k}_{0^{-}}$ standardization of neutron activation analysis;
Extensions, applications, prospects. Journal of Radioanalytical Chemistry, vol. 169, pp125-158.

Jonah, S.A.; Balogun, G.I.; Umar, I.M.; Mayaki, M. C. (2005): Neutron spectrum parameters in irradiation channels of the Nigeria Research Reactor-1 (NIRR-1) for the $\mathrm{k}_{0}$-NAA standardization. Journal of Radioanalytical and Nuclear Chemistry, Vol. 266, No. 1, p 83-88.

Kennedy, G.; St-Pierre, J.; Wang, K.; Zang, Y.; Preston, J.; Grant, C.; Vutchkov, M. (2000): Activation constants for Slowpoke and MNS reactors calculated from the neutron spectrum and $\mathrm{k}_{0}$ and $\mathrm{Q}_{0}$ values. Journal of Radioanalytical and Nuclear Chemistry, Vol. 245, No. 1, pp167-172.

Wang Liyu, (2004): WINSPAN 2004. A Multi Purpose Gamma-Ray Spectrum Analysis Software, CIAE, Beijing, China. 